\title{
Bisico stellt vor: Algi-Press, der Alginat-Ersatz für Situationsabformungen und mehr
}

\begin{abstract}
Hohe Dimensionsstabilität und Lagerfähigkeit
Die Abformung hat eine deutlich höhere Präzision, kann über Wochen eingelagert werden und ist mehrfach ausgießbar. Auch Wochen nach der Abformung bleibt der Abdruck unverändert und entspricht der oralen Situation des Patienten. Im Fall des Bruchs eines Provisoriums kann der Abdruck wiederverwendet werden. Eine doppelte Abformung ist nicht notwendig.
\end{abstract}

\section{Hervorragende Materialeigenschaften}

Eine ausreichende Verarbeitungszeit, kurze Mundverweildauer und der angenehme Pfefferminzgeschmack machen die Abformung für Patienten und Zahnarzt oder Mitarbeiterin angenehm. Algi-Press fließt dank der starken Hydrophilie sehr gut an. Die hohe Druckfestigkeit und Elastizität ermöglichen eine leichte Entnahme des Abdrucks. Ein weiterer Vorteil der Silikone gegenüber Alginaten ist die glatte Oberfläche von Modellen und Provisorien. Dies minimiert den Polieraufwand. Die Abdrücke müssen nicht feucht gelagert werden und können problemlos desinfiziert werden.

\section{Einfache Handhabung}

Die Handhabung ist dank der 380-Milliliter-Kartusche extrem einfach und sauber.

Die Mischung erfolgt nicht per Hand, sondern automatisch, staubfrei und schnell im Sympress (Renfert) oder anderen Mischgeräten. Die Dosierung ist immer exakt und homogen. Algi-Press garantiert sicheres und effizientes Arbeiten im Praxisalltag.

Als besonderen Service bieten bisico und Dynadent - Service für Zahnärzte GmbH den Verbandsmitgliedern ein exklusives Einführungsangebot:

\section{Algi-Press \\ - 1 Kartusche à $380 \mathrm{ml}$ \\ + 10 Mischer 5:1 \\ $\checkmark+1$ Bajonettring}

\section{Einführungspreis: 25 Euro statt 41 Euro}

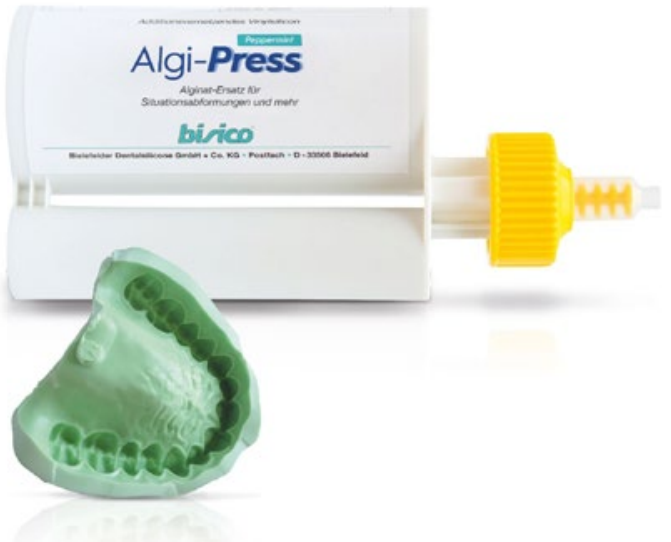

Mitglieder des Freien Verbandes Deutscher Zahnärzte erhalten zusätzlich zu ihrer Probebestellung:

\section{Mini-Spritze Provi Temp K}

Kunststoffmaterial auf Basis multifunktioneller Acrylate zur Herstellung provisorischer Kronen und Brücken.

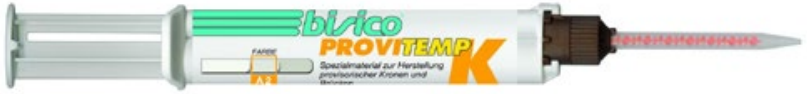

\section{Provi Bond K}

Eugenolfreier, temporärer Befestigungszement für provisorische Kronen und Brücken.

im Gesamtwert von 44,80 Euro gratis dazu.

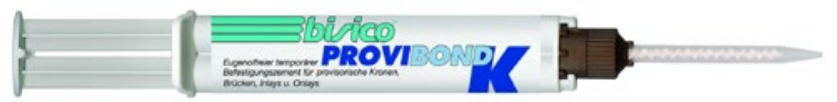

Nutzen Sie den Service-Coupon auf Seite xx für Ihre Bestellung oder besuchen Sie www.dynadent.de, hier finden Sie auch die gesamte Produktpalette von bisico.

Alle Preise zzgl. MwSt., zzgl. 4,20€ Versandkosten 